\section{OPEN ACCESS}

Edited by:

Heather Francis,

Indiana University, United States

Reviewed by:

Tianhao Zhou,

Indiana University, United States

Debjyoti Kundu,

Indiana University School of Medicine,

United States

${ }^{*}$ Correspondence:

Jia-bo Wang

jiabo_wang@ccmu.edu.cn

Yue-cheng Yu

gslsycy@163.com

Zheng-sheng Zou

zszou302@163.com

Specialty section: This article was submitted to

Gastroenterology,

a section of the journa

Frontiers in Medicine

Received: 21 December 2020

Accepted: 22 February 2021

Published: 12 March 2021

Citation:

Li S-s, Niu M, Jing J, Huang Y, Zhang Z-t, Chen S-s, Shi G-Z, He X,

Zhang H-z, Xiao X-h, Zou Z-s, Yu Y-c

and Wang J-b (2021) Metabolomic Signatures of Autoimmune Hepatitis in

the Development of Cirrhosis.

Front. Med. 8:644376.

doi: 10.3389/fmed.2021.644376

\title{
Metabolomic Signatures of Autoimmune Hepatitis in the Development of Cirrhosis
}

\begin{abstract}
Shan-shan $\mathrm{Li}^{1,2}$, Ming $\mathrm{Niu}^{3}$, Jing Jing ${ }^{2}$, Ying Huang ${ }^{2}$, Zi-teng Zhang ${ }^{2}$, Shuai-shuai Chen ${ }^{2}$, Ge-zi Shi ${ }^{2}$, Xian He ${ }^{1,2}$, Hai-zhu Zhang ${ }^{1}$, Xiao-he Xiao ${ }^{2}$, Zheng-sheng Zou ${ }^{2 *}$, Yue-cheng Yu ${ }^{4 *}$ and Jia-bo Wang ${ }^{2,5 *}$

${ }^{1}$ School of Pharmacy and Chemistry, Dali University, Dali, China, ${ }^{2}$ Department of Liver Diseases, Fifth Medical Center of Chinese PLA General Hospital, Beijing, China, ${ }^{3}$ Department of Poisoning Treatment, Fifth Medical Center of Chinese PLA General Hospital, Beijing, China, ${ }^{4}$ Liver Diseases Center of General Hospital of PLA Eastern Theater Command, Bayi Hospital Affiliated to Nanjing University of Chinese Medicine, Nanjing, China, ${ }^{5}$ School of Traditional Chinese Medicine, Capital Medical University, Beijing, China
\end{abstract}

Objectives: Autoimmune hepatitis $(\mathrm{AlH})$ can progress into severe outcomes, i.e., decompensated cirrhosis, from remarkable and persistent inflammation in the liver. Considering the energy-expending nature of inflammation, we tried to define the metabolomics signatures of $\mathrm{AlH}$ to uncover the underlying mechanisms of cirrhosis development and its metabolic biomarkers.

Methods: Untargeted metabolomics analysis was performed on sera samples from 79 AlH patients at the stages (phenotypes) of non-cirrhosis $(n=27)$, compensated cirrhosis $(n=22)$, and decompensated cirrhosis $(n=30)$. Pattern recognition was used to find unique metabolite fingerprints of cirrhosis with or without decompensation.

Results: Out of the 294 annotated metabolites identified, 2 metabolic fingerprints were found associated with the development of cirrhosis (independent of the decompensated state, 42 metabolites) and the evolution of decompensated cirrhosis (out of 47 metabolites), respectively. The cirrhosis-associated fingerprints (eigenmetabolite) showed better capability to differentiate cirrhosis from non-cirrhosis patients than the aminotransferase-to-platelet ratio index. From the metabolic fingerprints, we found two pairs of metabolites (Mesobilirubinogen/6-Hydroxynicotinic acid and LysoPA(8:0/0:0)/7alpha-Hydroxycholesterol) calculated as ratio of intensities, which revealed robust abilities to identify cirrhosis or predict decompensated patients, respectively. These phenotype-related fingerprint metabolites featured fundamental energy supply disturbance along with the development of $\mathrm{AlH}$ cirrhosis and progression to decompensation, which was characterized as increased lipolysis, enhanced proteolysis, and increased glycolysis.

Conclusions: Remodeling of metabolism to meet the liver inflammation-related energy supply is one of the key signatures of $\mathrm{AlH}$ in the development of cirrhosis and decompensation. Therefore, drug regulation metabolism has great potential in the treatment of $\mathrm{AlH}$.

Keywords: liver cirrhosis, autoimmune hepatitis, metabolomics, metabolic pathway, biomarkers 


\section{INTRODUCTION}

Autoimmune hepatitis (AIH) is an inflammatory disease of the liver mediated by an abnormal autoimmune response (1). The pathogenesis of AIH is not fully understood. AIH patients may have long-term asymptomatic tissue inflammation, but they are often not diagnosed, thus losing treatment opportunities. When clinical symptoms appear, the disease may have progressed to a more severe stage, and may even further progress to cirrhosis, decompensation of liver function and eventually liver failure $(2,3)$. AIH cirrhosis has no specific clinical manifestations in the compensated stage; When the disease progresses to the decompensated stage, liver dysfunction and portal hypertension will appear. Decompensated patients may also experience symptoms such as gastrointestinal bleeding, liver ascites, and coma. Liver transplantation is an effective treatment for decompensated patients (4), but AIH may recur after transplantation. Although AIH is a disease mediated by autoimmune abnormalities and autoantibodies are important for the diagnosis of $\mathrm{AIH}$, serum autoantibodies do not reflect the severity of the disease in AIH patients (5). Looking for biomarkers that can predict the progression of liver cirrhosis and malignancy in AIH patients is of immense significance in the clinical prognosis and understanding of the underlying mechanism of disease progression.

$\mathrm{AIH}$ is mainly characterized by liver inflammation, which is essentially a chronic and unresolved liver inflammatory damage (6). Inflammation is an energy-expensive biological process that requires a large amount of energy and intermediary metabolites for the synthesis of inflammatory factors and immune response (7). Continuous hepatic inflammation inevitably causes significant metabolic changes and induces a wide range of catabolic dysfunction, which may be involved in the progression of the disease. The liver is the main metabolic organ, responsible for most of the body's metabolic processes such as synthesis, decomposition, and transformation (8), thus, it is very important to study AIH from the perspective of metabolism.

Metabolomics is a phenotypic method for studying metabolites, small molecule substrates, and intermediary metabolites, which directly reflects potential biochemical activity and state of cells/tissues (9). Furthermore, it is a powerful method to explore specific biomarkers of liver diseases (10). In recent years, non-targeted metabolomics has been applied to the study of AIH-related biomarkers (11-13), but there is no research related to disease progression of $\mathrm{AIH}$.

Here, we report the findings of untargeted and whole spectrum metabolomic study in sera from $79 \mathrm{AIH}$ patients and compare the metabolic markers of liver cirrhosis and decompensation. The aim of this study is to characterize the sera metabolite fingerprints specific to patients with compensated or decompensated AIH cirrhosis, and to identify potential prognostic biomarkers of AIH.

\section{MATERIALS AND METHODS}

\section{Study Design}

We explored the sera samples of hospitalized AIH patients in the Biobank of Fifth Medical Center of Chinese PLA
General Hospital from October 31st, 2015 to May 19th, 2017. Patients diagnosed with $\mathrm{AIH}$ and where adequate clinical information was available, were selected for the study. This research scheme was approved by the Ethics Committee of the hospital based on the ethical principles of the Declaration of Helsinki, and written informed consent was obtained from all patients. Their sera had to be deposited within 4 days after diagnosis. Patients with competitive etiologies were excluded. There are $89 \mathrm{AIH}$ patients initially screened, and 10 of them were excluded due to competitive etiologies. Among those 10 excluded patients, 8 had concurrent drug-induced liver injury and 2 had alcoholic liver disease. Finally, 79 patients were enrolled in the study. The enrolled samples were divided into three groups: non-cirrhosis (NC) $(n=27)$, compensated cirrhosis (CC) $(n=22)$, and decompensated cirrhosis (DC) $(n=30)$ groups, according to the international clinical guidelines (14). Based on untargeted metabolomics, we acquired numerous metabolites (metabolome profile) with significant difference $(P<0.05$ and $\mathrm{FC}>1.5$ or $<0.67)$ by comparing $\mathrm{CC}$ and $\mathrm{DC}$ with $\mathrm{NC}$ and marked them as $(\mathrm{CC}+\mathrm{DC}) / \mathrm{NC}$. Metabolites identified in metabolome profiles were considered as ones with cirrhosis-associated metabolome features. Similarly, by comparing CC or DC with $\mathrm{NC}$, they were marked as CC/NC and $\mathrm{DC} / \mathrm{NC}$, respectively. The excluded part of DC/NC can be considered as decompensation-associated metabolome features. Also, hierarchical clustering was used to screen out the metabolic fingerprints, which consisted of a cluster of metabolites with high area under curve (AUC) of the receiver operating characteristic (ROC) analysis and $P$-values, in differentiating each group (phenotype). Furthermore, the phenotype-associated fingerprint metabolites were projected to one eigenmetabolite by reducing the dimension, which is defined as the first principal component in unsupervised principal component analysis (PCA) (15). The eigenmetabolite was further investigated to discover relationships with demographics, biochemistry, complications, and clinical evaluation models. Finally, metabolic pathways of phenotypeassociated fingerprint metabolites were analyzed to interpret the underlying mechanisms of the metabolic drivers in the evolution of AIH.

\section{Samples Preparation and Chromatographic Conditions}

The biobanked serum were processed according to the literature (16). Quality control samples (QC) were prepared by mixing $10 \mu \mathrm{L}$ from each sample to be analyzed. Chromatographic column: ZORBOX RRHD C18 analytical column (2.1 mm i.d. $\times 100 \mathrm{~mm}, 1.8 \mu \mathrm{m}$ i.d., Agilent Technologies, USA). Column temperature: $30^{\circ} \mathrm{C}$. Sample temperature: $4^{\circ} \mathrm{C}$. The mobile phase was composed of solvent A: Water with $0.1 \%$ formic acid in positive mode of Q-TOF, pure water in negative mode, mobile phase B: Acetonitrile. Flow rate: $0.30 \mathrm{~mL} / \mathrm{min}$; Sample injection size: $4 \mu \mathrm{L}$. Chromatographic gradient elution conditions: 0$1 \mathrm{~min}, 100 \%$ (A); 1-9 min, 100\%-60\% (A); 9-19 min, 60-10\% (A); 19-21 $\mathrm{min}, 10-0 \%$ (A); 21-25 $\mathrm{min}, 100 \%$ (B).

\section{Mass Spectrum Condition}

An Agilent 6550 Q-TOF LC/MS with an electrospray ionization source (ESI) in positive and negative ion modes was used. 
TABLE 1 | Clinical information comparison between liver disease patients with NC, CC, and DC.

\begin{tabular}{|c|c|c|c|c|c|}
\hline & Non-cirrhosis $(n=27)$ & Compensated cirrhosis $(n=22)$ & Decompensated cirrhosis $(n=30)$ & $P^{a}$ & $P^{b}$ \\
\hline Age/year & $51(42,56)$ & $54(50,63)$ & $57.5(51.0,67.0)$ & 0.224 & 0.274 \\
\hline Female, $n(\%)$ & $27(100.0)$ & 18(81.8) & 28(93.3) & - & - \\
\hline AST/U. $L^{-1}$ & $59.0(26.0,121.0)$ & $75.0(30.8,171.5)$ & $49.5(36.8,117.8)$ & 0.148 & 0.788 \\
\hline AST/ALT & $1.4(1.1,2.2)$ & $1.6(0.9,2.4)$ & $1.8(1.3,2.7)$ & 0.702 & 0.404 \\
\hline $\mathrm{TBil} / \mathrm{mg} \cdot \mathrm{dl}^{-1}$ & $14.2(10.2,22.4)$ & $22.3(13.5,61.4)$ & $26.6(11.8,83.5)$ & 0.03 & 0.624 \\
\hline $\mathrm{DBil} / \mu \mathrm{mo} \cdot \mathrm{L}^{-1}$ & $5.7(3.3,12.0)$ & $11.6(5.3,47.1)$ & $14.5(5.4,66.5)$ & 0.027 & 0.657 \\
\hline $\lg A / g \cdot L^{-1}$ & $2.6(1.9,3.5)$ & $2.9(1.9,4.6)$ & $3.7(2.4,6.2)$ & 0.488 & 0.154 \\
\hline $\lg G / g \cdot L^{-1}$ & $15.0(12.1,21.3)$ & $21.6(15.5,24.0)$ & $20.4(16.2,25.8)$ & 0.062 & 0.97 \\
\hline $\operatorname{lgM} / g \cdot L^{-1}$ & $1.2(0.8,1.7)$ & $1.7(0.9,2.9)$ & $1.7(1.1,2.3)$ & 0.093 & 0.795 \\
\hline TG/mmol.L-1 & $1.1(0.9,1.5)$ & $1.3(0.8,1.7)$ & $1.0(0.8,1.6)$ & 0.96 & 0.535 \\
\hline ALB/g.L-1 & $36.0(34.0,40.0)$ & $33.0(29.5,37.0)$ & $29.5(25.8,33.3)$ & 0.025 & 0.05 \\
\hline $\mathrm{WBC} / \mathrm{mm}^{3}$ & $5140.0(4340.0,6800.0)$ & $3825.0(3155.0,4777.5)$ & $3850.0(2852.5,5010.0)$ & 0.005 & 0.926 \\
\hline $\mathrm{PLT} / 10^{9} \cdot \mathrm{L}^{-1}$ & 195.0(168.0,223.0) & $101.0(62.5,158.0)$ & $84.0(53.5,124.5)$ & $<0.01$ & 0.229 \\
\hline TBA/umol.L-1 & $22.0(7.0,37.0)$ & $35.5(17.3,97.8)$ & $47.0(16.0,108.3)$ & 0.033 & 0.853 \\
\hline Cr/umol.L-1 & $59.0(51.0,70.0)$ & $60.0(53.5,65.8)$ & $66.0(60.5,74.3)$ & 0.371 & 0.081 \\
\hline $\mathrm{PT} / \mathrm{s}$ & $11.2(10.6,11.7)$ & $12 \cdot 3(11.0,13.7)$ & $12.8(11.2,14.7)$ & 0.008 & 0.188 \\
\hline ANA & $1: 100(1: 486,1: 100)$ & $1: 320(1: 1,000,1: 100)$ & $1: 320(1: 1000,1: 100)$ & 0.102 & 0.669 \\
\hline$\gamma$-globulin/\% & $24.0(18.0,28.8)$ & $29.4(22.4,33.8)$ & $32.0(25.6,35.0)$ & 0.052 & 0.4 \\
\hline With ascites (\%) & $0(0.00)$ & $0(0.00)$ & $26(86.7)$ & - & - \\
\hline With HE (\%) & $0(0.00)$ & $0(0.00)$ & $0(0.00)$ & - & - \\
\hline
\end{tabular}

All clinical information in the table indicates data collected at the time closest to blood collection.

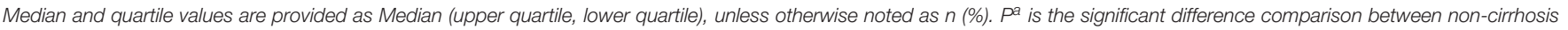
and compensated cirrhosis patients, $P^{b}$ between compensated and decompensated cirrhosis patients.

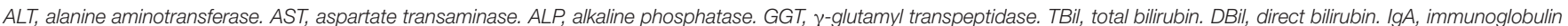

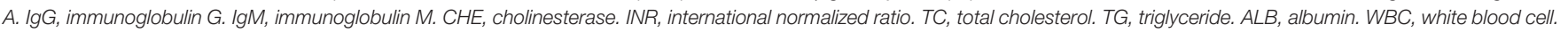
PLT, platelet. TBA, total bile acid. Cr, creatinine. PT, prothrombin time. ANA, antinuclear. HE, hepatic encephalopathy. APRI, AST to PLT ratio index.

Non-targeted primary mass spectrometric detection conditions: The mass range of $\mathrm{m} / \mathrm{z} 50-1,200$; gas temperature of $225^{\circ} \mathrm{C}$ in both positive and negative ionization modes; airflow of 13 $1 / \mathrm{min}$; the nebulizer of 20 pisg (negative) and 20 pisg (positive); sheath gas temperature of $275^{\circ} \mathrm{C}$ and sheath gas flow of $12 \mathrm{l} / \mathrm{min}$; electrospray capillary voltage of $3,500 \mathrm{~V}$ in negative ionization mode and 4,000 $\mathrm{V}$ in positive ionization mode; and nozzle voltage of $2,000 \mathrm{~V}$ in the positive and negative ion modes. The acquisition mode was ESI Continuum mode and 14,104 variables were captured in positive ion mode as well as 13,357 variables were captured in negative ion mode.

\section{Statistical Analysis}

All variables were normalized in MetaboAnalyst 4.0 (https:// www.metaboan-alyst.ca) after being preprocessed in Masshunter Profinder software. Summarized results of the patients' clinical biochemical characteristics for continuous variable are expressed as median (upper quartile, lower quartile), and categorical variables are expressed as numbers and percentage. Comparisons between groups were made with Kruskal-Wallis $\mathrm{H}$ test. All statistical analysis was performed using SPSS 21.0 software. The significance level for all statistical tests was set at 0.05 . PCA and orthogonal partial least squares discrimination analysis (OPLS-DA) were performed in SIMCA -P 14.1 software. The Pvalues, fold change (FC) and AUC of ROC were obtained from the MetaboAnalyst website. We narrowed down the original variables to fingerprint by $P$-value, FC, and AUC.

\section{RESULTS}

\section{Baseline Demographic and Clinical Characteristics of the Study Cohort}

The baseline demography and clinical characteristics among NC, CC, and DC groups are described in Table 1. There was no difference in the mean ages among the three groups. Except for 6 male patients, female patients accounted for an overwhelming proportion of $\mathrm{AIH}$ patients $(n=73,92.41 \%)$. Between $\mathrm{NC}$ 
and CC groups, the levels of TBA, PT, and INR in CC were much higher than those in NC, and the levels of CHE, ALB, PLT, and WBC in CC were significantly lower than those in NC. Significantly lower levels of ALB and CHE were found in DC groups as compared to the CC group. With respect to complications, $86.67 \%$ of patients in the DC group had ascites. Besides, there was no hepatic encephalopathy in AIH patients. Aminotransferase-to-platelet ratio index (APRI) score showed an increasing trend along with the development of cirrhosis from $\mathrm{NC}$ to CC and DC.

\section{Patients With Cirrhosis Exhibited Different Anabolism and Metabolism Characteristics} Compared to non-cirrhotic AIH patients, those that developed into the cirrhosis stage (CC or DC) showed different characteristics, including sera enzymes, liver-synthesizing proteins, liver-metabolizing metabolites, and coagulation function (Table 1). When cirrhosis developed, significant decrease in albumin and white blood cell counts were observed. The coagulation function indices (prothrombin time and international normalized ratio) increased significantly, but were not significant between CC and DC groups. Bilirubin (TBiL and $\mathrm{DBiL}$ ) increased significantly in the CC group compared to the NC group, indicating potential liver dysfunction of detoxication. Along with the evolving disease stages, cholinesterase decreased significantly. Together, these results indicated a gradual disturbance of liver functions such as anabolism and metabolism in the evolution of AIH.

\section{Liver Cirrhosis and Decompensation Stages Associated With Different Sera Metabolites}

We observed significant alterations in the metabolomic profiles from either positive or negative modes of mass spectrometry, in different stages of AIH (Figures 1A-D). This indicates that the progression from $\mathrm{NC}$ to $\mathrm{CC}$ and $\mathrm{DC}$ was a major contributor for the alteration of the sera metabolome. To explore the underlying relationship between the evolving stages of $\mathrm{AIH}$ and the patterns of sera metabolite levels, we firstly obtained metabolic entities with significant difference $(P<0.05)$ between NC and CC, as well as CC vs. DC. The Venn diagram of these metabolic entities showed over 1,200 metabolites (including both positive and negative modes) that were associated with cirrhosis [(CC+DC) vs. NC]; while over 1,600 metabolites were solely associated with decompensation (DC vs. CC) (Figures 1E,F). We then identified 124 and 170 metabolites in these two sets of variables, respectively. Based on these metabolites, we found that the PCA plot of 124 cirrhosis-related metabolites showed an obvious separation of NC from either CC or DC, while CC and DC overlapped (Figure 1G). The PCA plot of the 170 decompensation-related metabolites revealed an evident separation of DC from either CC or NC, while CC and NC were located in the same zone (Figure $\mathbf{1 H}$ ). These results indicate two distinct metabolic features associated with cirrhosis or decompensation stages. The fold changes of these annotated metabolites between the study groups are showed in Supplementary Figure 1.

\section{Cirrhosis-Associated Sera Metabolites Composed of a Fingerprint for AIH-Related Liver Cirrhosis}

To reduce the dimension of the data set and find a unique metabolomic fingerprint of AIH-related liver cirrhosis, we computed the AUC and $P$-values of each of the 124 cirrhosisrelated metabolites in differentiating cirrhosis (either CC or DC) from non-cirrhosis (NC). The resulting AUCs and associated $P$ values were used for a hierarchical cluster analysis and shown in a heat-map, indicating the highest relevant cluster (including 42 metabolites) with a highly significant association with cirrhosis [Supplementary Figure 2(I)]. The AUC values in this cluster for cirrhosis ranged from 0.6786 to 0.7835 with significant $P$-values (Supplementary Tables 1, 3). Interestingly, the 42 metabolite cluster identified in Supplementary Figure 2 (I), the high AUC values observed in cirrhosis (either CC or DC) were maintained when patients were separated into CC or DC groups [Supplementary Figure 2(II,III)]. These results indicate a unique metabolome fingerprint (42 metabolites) among patients with cirrhosis.

To further display the metabolome fingerprint trend in AIH development, we computed an eigenmetabolite (15) using PCA as a representative variable of the aforementioned 42metabolite cluster. The eigenmetabolite showed progressively decreasing trend across the different stages of $\mathrm{AIH}$ and reached a minimum in DC (Figure 2A). Interestingly, there was a significant decrease of the eigenmetabolite in CC compared to NC, but no significance was observed between CC and DC. This trend supports the result that the 42 metabolites represented a metabolic fingerprint for AIH-related liver cirrhosis.

\section{Cirrhosis-Associated Metabolic Fingerprint Showed Better Diagnostic Performance in Discerning AlH Cirrhosis Than That of APRI} We found that the cirrhosis-associated metabolic fingerprint (eigenmetabolite) had better capability of identifying cirrhotic from non-cirrhotic patients than APRI (AUC values 0.874 vs. 0.763, Figures 2B,C). Collectively, these findings indicate that the 42-metabolite cluster can serve as a fingerprint of sera metabolites, which characteristically differentiated cirrhosis from non-cirrhosis.

\section{Decompensation-Associated Sera Metabolites Composed a Fingerprint for Differentiating Decompensation Stage}

Similarly, for each of the 170 metabolites in decompensationassociated metabolome feature, we computed the AUC assessing the differentiation accuracy of each metabolite in decompensated cirrhosis from the compensation stage. The resulting AUCs and associated $P$-values were used for a hierarchical cluster analysis, which identified the highest relevant cluster including 47 metabolites with a highly significant association with cirrhosis (AUC for cirrhosis and significant $P$ shown in 


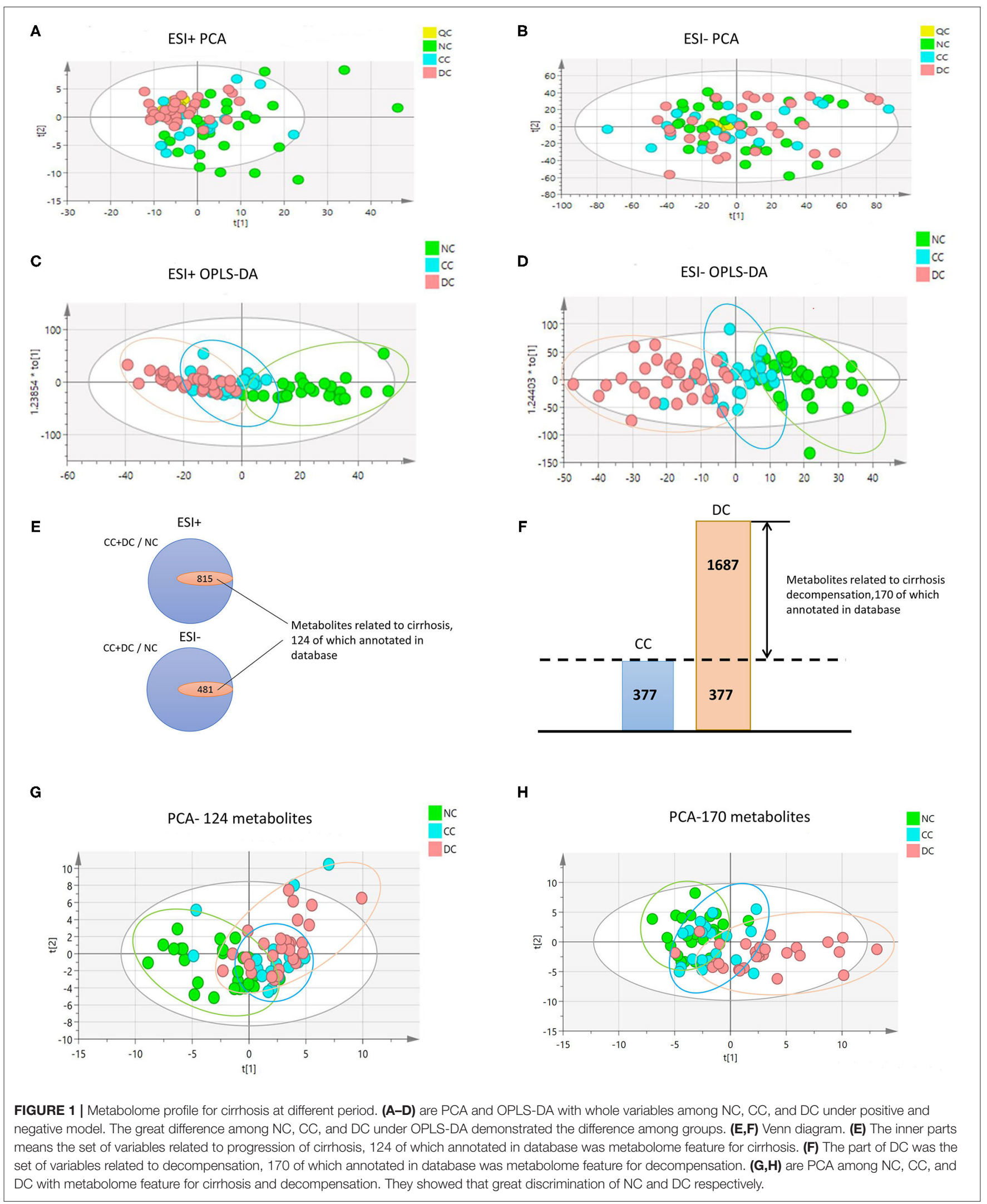




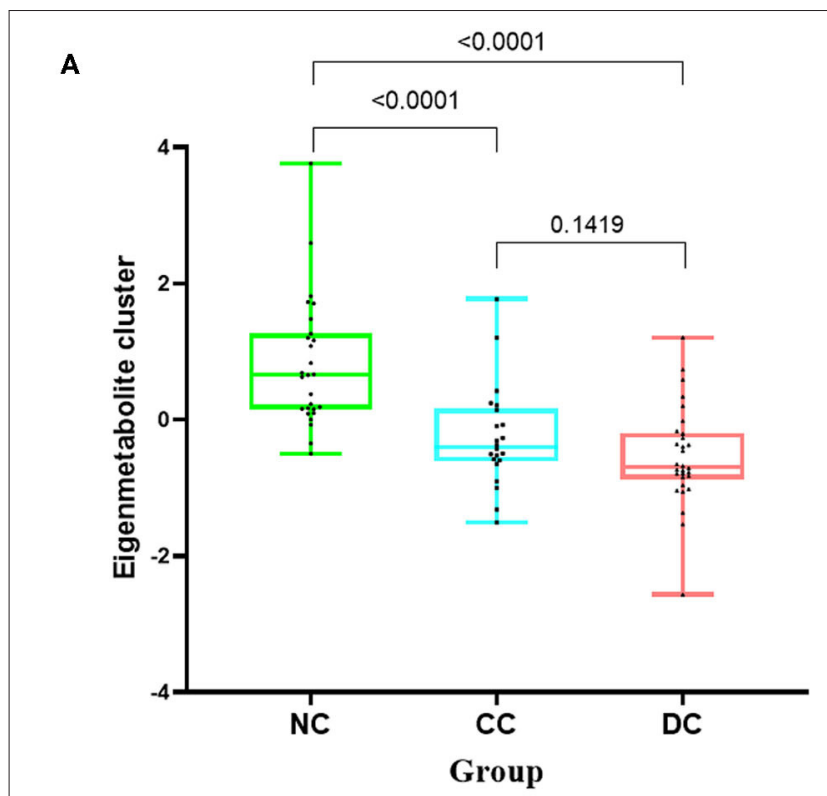

c

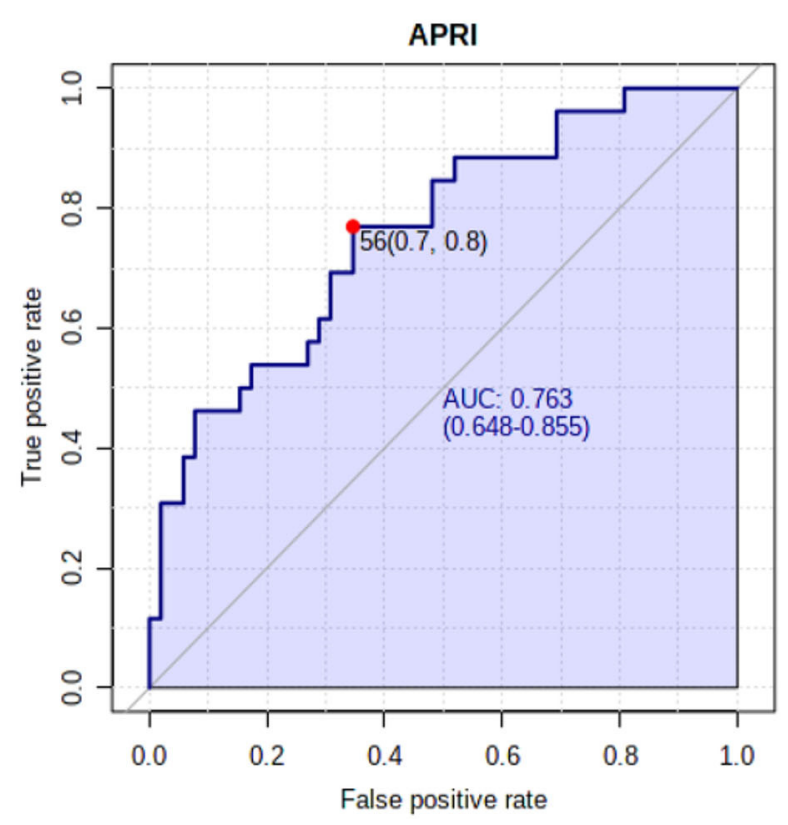

B

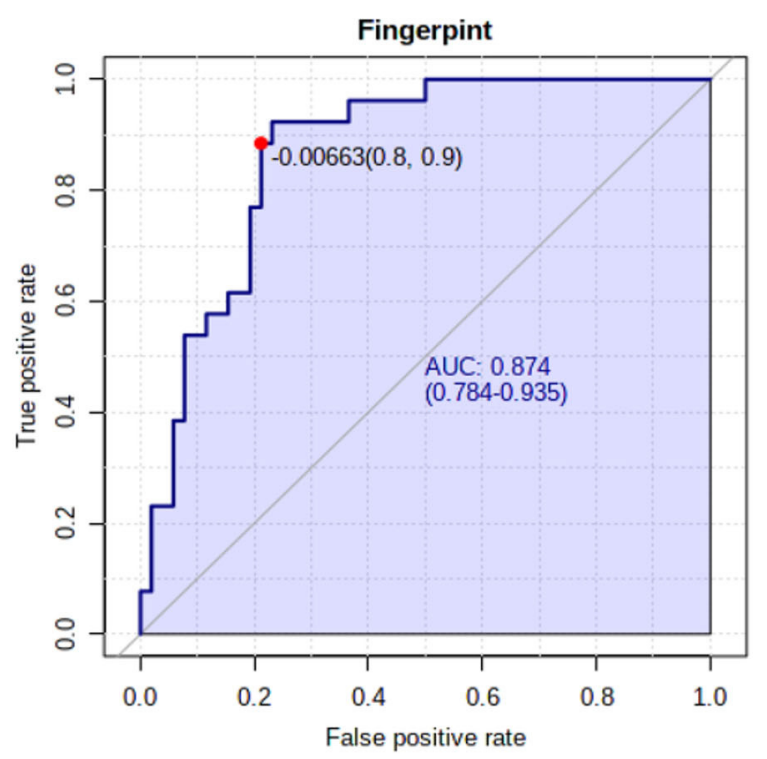

D

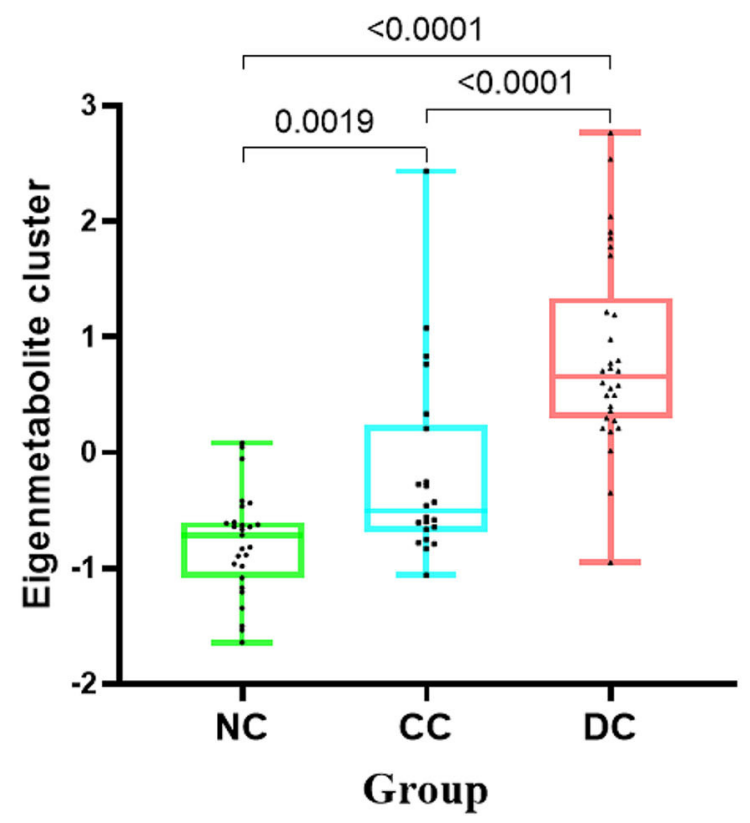

FIGURE 2 | Identification of cirrhosis-related metabolome fingerprint. (A) Showed the decreasing trend of eigenmetabolite of metabolome fingerprint along with the progression of cirrhosis. (B) Demonstrated the cirrhosis-associated metabolic fingerprint (eigenmetabolite) revealed better capability to identify cirrhotic patients from non-cirrhotic ones(AUC:0.874). (C) The ROC curve of APRI in discriminating cirrhosis from non-cirrhotic AlH patients(AUC:0.763). (D) Showed that the increasing trend of eigenmetabolite of metabolome fingerprint from NC to CC and DC.

[Supplementary Figure 3, Supplementary Table 2]). We then computed an eigenmetabolite by PCA, which is representative of the 47-metabolite cluster and correlated the eigenmetabolite with the progression of AIH, from NC to CC and DC (Figure 2D). The eigenmetabolite showed an increasing trend and significant differences (all $P<0.05$ ) among NC, CC, and DC.

\section{Metabolic Fingerprints Indicating Loss of Liver Anabolic Functions}

We further compared trends of the two metabolic fingerprints separately using clinically important indices $(14,17,18)$. Cirrhosis-associated eigenmetabolite were significantly higher in NC and CC patients with high-albumin (Figure 3A), which 
A

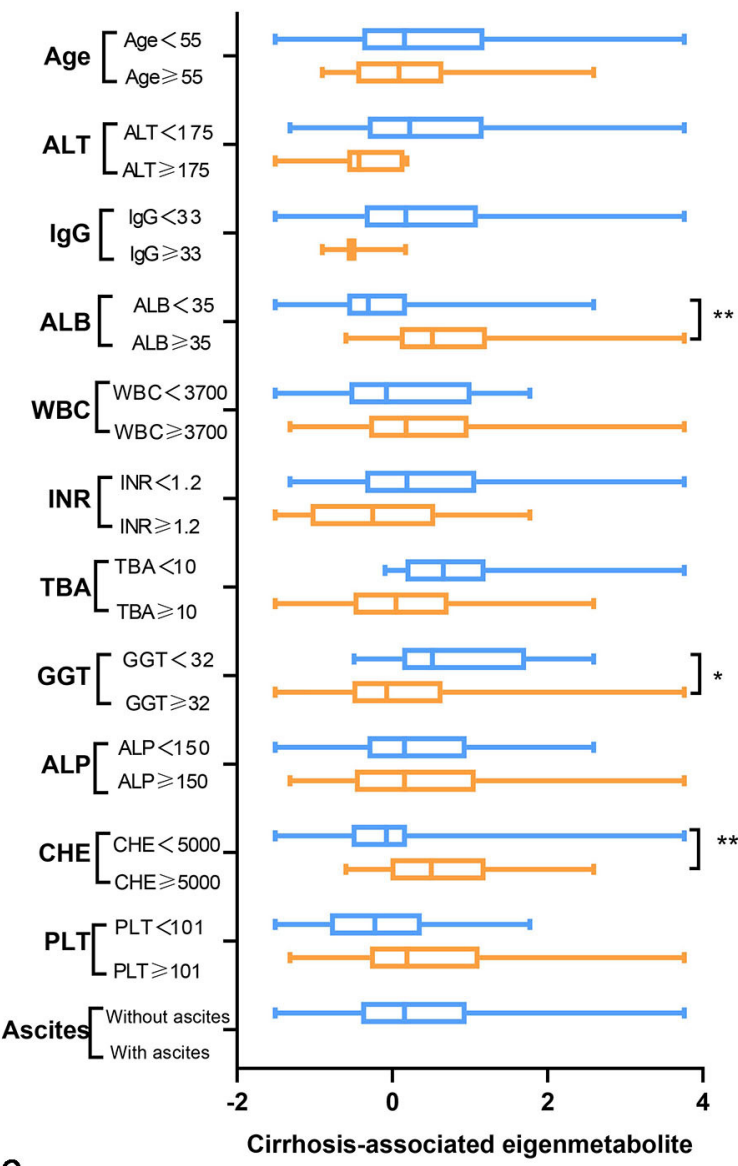

C

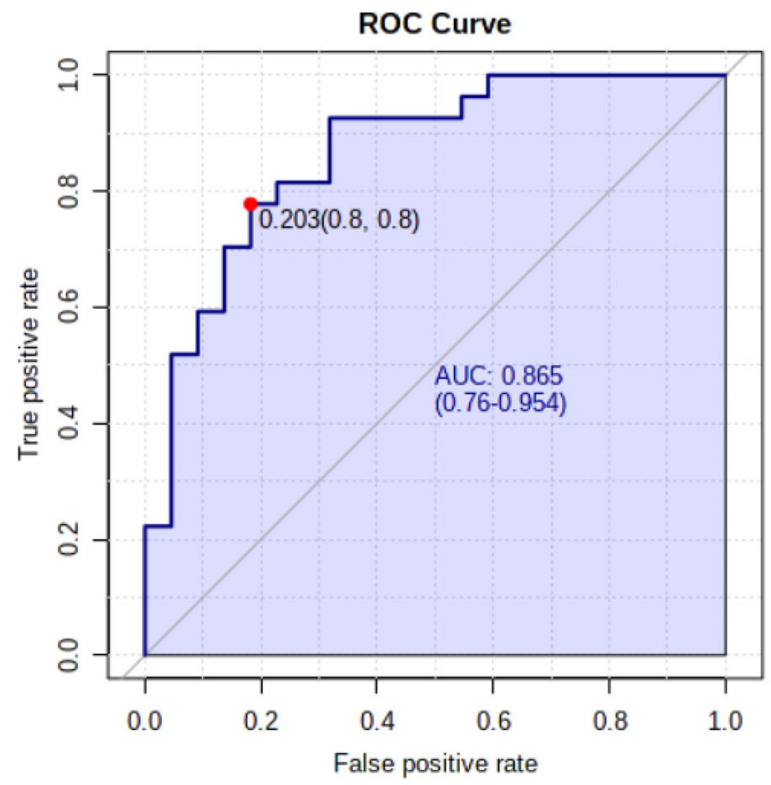

Mesobilirubinogen and 6-Hydroxynicotinic acid

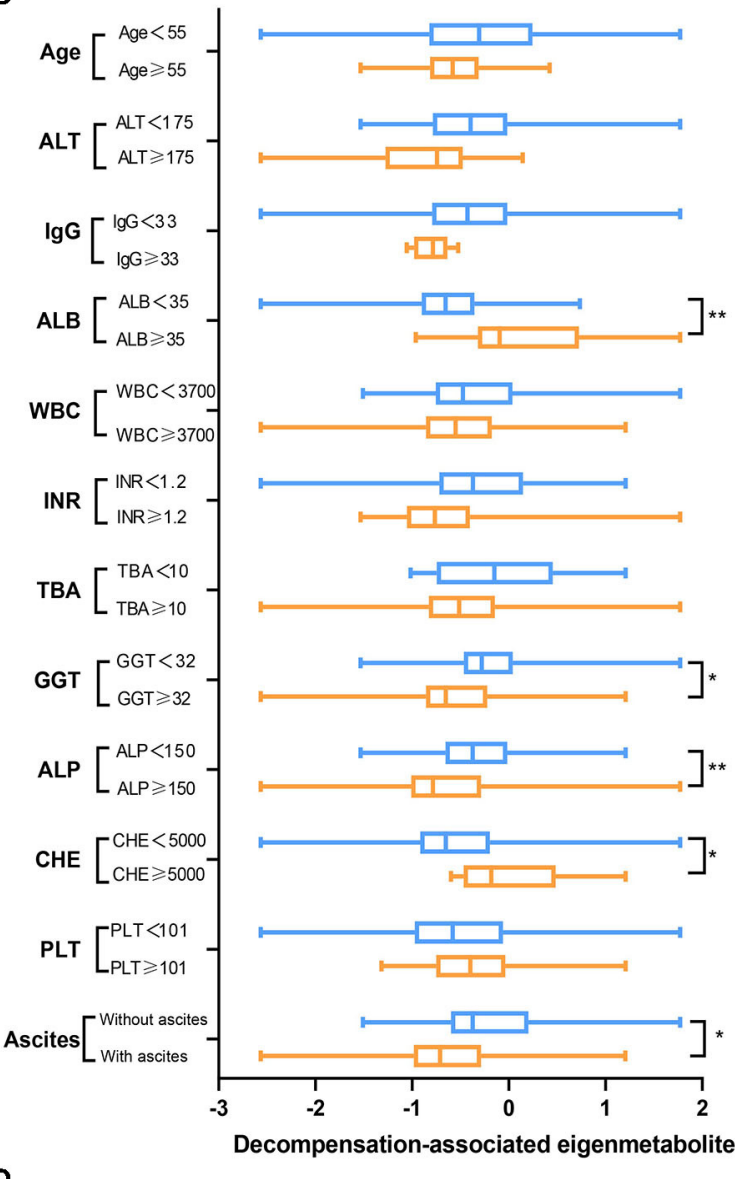

D

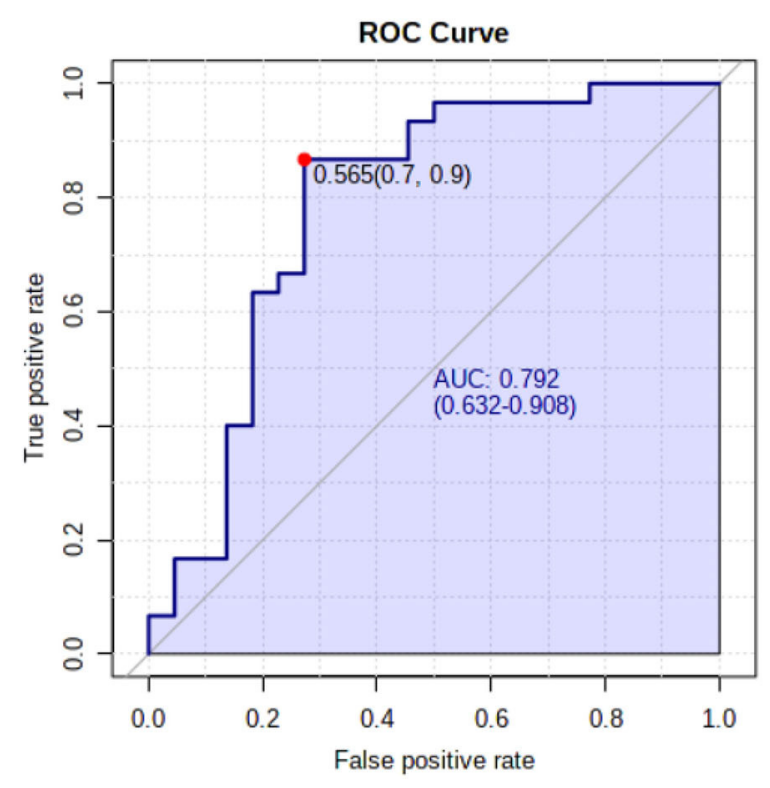

LysoPA(8:0/0:0) and 7alpha-Hydroxycholesterol

FIGURE 3 | Clinical associations of the two metabolic fingerprints and the diagnostic ability of paired metabolites. (A) Comparison between clinical indices and the cirrhosis-associated fingerprint (eigenmetabolite) in NC and CC patients. (B) Comparison between clinical indices and the decompensation-associated fingerprint (eigenmetabolite) in CC and DC patients. (C) The diagnosis ability of intensity ratio between Mesobilirubinogen and 6-Hydroxynicotinic acid to differentiate NC and CC. (D) The diagnosis ability of intensity ratio between LysoPA(8:0/0:0) and 7alpha-Hydroxycholesterol to differentiate CC and DC(The units of each index are indicated in Table 1). 
indicates the loss of liver anabolic function in cirrhotic AIH patients. Albumin-associated scores may be useful to evaluate long-term prognosis in patients with autoimmune-related hepatic cirrhosis (19). Similarly, high level of eigenmetabolite was observed in patients with high cholinesterase. In some clinical indices, decompensation-associated metabolic fingerprint in CC and DC patients had significant alterations in their subgroups (Figure 3B). Notably, significant $P$-value also existed between patients with low-albumin and high-albumin indicating further loss of liver anabolic functions in decompensated cirrhotic patients. In addition, high level of alkaline phosphatase corelated with low level of decompensation-associated eigenmetabolite, indicating low anabolic liver function $(20,21)$. Besides, low levels of eigenmetabolite were observed in patients with high $\gamma$-glutamyl transpeptidase in cirrhosis and decompensated patients. Gamma-glutamyl transpeptidase and albumin were independent predictors of significant fibrosis $(22,23)$. In cirrhotic patients with ascites, low level of decompensation-associated eigenmetabolite was observed compared to non-ascites patients.

\section{Paired Metabolites Reveal Diagnostic and Prognostic Capability for AlH}

From the cirrhosis-associated fingerprint, a metabolites pairMesobilirubinogen and 6-Hydroxynicotinic acid-was further screened out to construct a novel diagnostic parameter to identify AIH-related cirrhosis patients (Figure 3C). The diagnostic ability of such paired metabolites is based on the intensity ratio, which was 0.865 of AUC. The intensity ratio of paired metabolites can eliminate the differences between instruments and provide better methodology and good applicability for clinical use. Similarly, another metabolites pair-LysoPA(8:0/0:0) and 7alpha-Hydroxycholesterol-was screened out from the decompensation-associated fingerprint and achieved 0.792 of AUC in differentiating decompensated cirrhosis from nondecompensated ones (Figure 3D).

\section{Metabolic Fingerprints Have Potential Pathophysiological Significance in the Evolution of AlH Cirrhosis}

Several energy sources and intermediary metabolites in the plasma of patients with liver cirrhosis changed significantly compared to non-cirrhosis patients, which indicates a particularly significant energy metabolism disorder. We found that the two metabolic fingerprints are obviously related to the energy supply related to the interference of metabolic pathways, especially increased lipolysis and proteolysis (Figure 4). These results indicate that abnormalities in metabolic fingerprints may be involved in the progression of the disease.

\section{AlH Cirrhosis Associates With Increased Lipolysis}

In the two metabolic fingerprints for different stages of $\mathrm{AIH}$, a typical feature is that a series of metabolites related to long-chain fatty acids (e.g.,12,13-DHOME, trans-2-EnoylOPC4-CoA, Docosadienoyl-CoA, Tetrac-osatetraenoyl-CoA and (S)-3-Hydroxydodecanoyl-CoA, etc.) are significantly reduced (Supplementary Tables 1, 2). Increased lipolysis and suppressed lipogenesis strikingly promote the energy supplying flux by fatty acids.

\section{AlH Cirrhosis Associates With Increased Proteolysis}

Due to a large amount of energy expenditure, a key feature of decompensated liver cirrhosis was observed to be used as energy supply by proteolysis (Figure 4). Several proteolysis markers Glutaminylphenylalanine, Hydroxyprolyl-Tyrosine, and Glutaminyltryptophan, and incomplete breakdown products of protein catabolism, increased significantly in DC group compared to the NC or CC groups (Supplementary Table 3).

\section{DISCUSSION}

This paper is the first study utilizing untargeted and wholespectrum metabolomics approach in sera samples of AIH patients without cirrhosis or with cirrhosis at either compensation or decompensation stages. We identified two metabolic fingerprints for different stages of AIH. The fingerprint of metabolites related to liver cirrhosis includes 42 metabolites, reflecting the characteristic metabolic changes in the process of $\mathrm{AIH}$ progression to liver cirrhosis, without distinguishing between compensatory and decompensated states. Compared to non-invasive liver cirrhosis evaluation index APRI, liver cirrhosis-related metabolite fingerprints have a better diagnostic ability to distinguish between non-cirrhosis and liver cirrhosis. The metabolic fingerprint related to decompensation includes 47 metabolites, reflecting the characteristic metabolic changes from cirrhosis to the decompensated stage. These metabolic characteristics provide a new perspective to understand the underlying mechanism of AIH disease progression. Specifically, the analysis of pathway enrichment by combining two metabolic fingerprints indicates that $\mathrm{AIH}$ progresses to liver cirrhosis and decompensated stage, which is manifested by obvious disturbances in metabolic processes related to energy supply, and nutrient metabolism is the basic characteristic of decomposition and consumption. These metabolic characteristics are further strengthened from compensated cirrhosis to decompensated cirrhosis.

AIH-related immune response may be caused by the autoantigens of initial CD4+ T cells (24), which further forms continuous immune response and inflammatory reaction. There are many studies on the relationship between immunology and metabolism (25-27), but little is known about the metabolic adaptation and characteristics of $\mathrm{AIH}$ disease progression. $\mathrm{AIH}$ is mainly characterized by liver interface inflammation. As the disease progresses, it produces energy dependence, leading to energy competition between the immune system and other programs in the body. At the same time, the energy supply in the body is obviously disordered, and nutrients (such as lipids and proteins) are consumed in large quantities (7), which manifests as increased lipolysis and proteolysis. In this study, we found that a group of pathways involved in energy metabolism constituting the metabonomic characteristics of AIH. L-kynurenine and its further breakdown products carry out diverse biological functions, including dilating blood vessels during inflammation and regulating the immune response $(28,29)$. The elevation of 

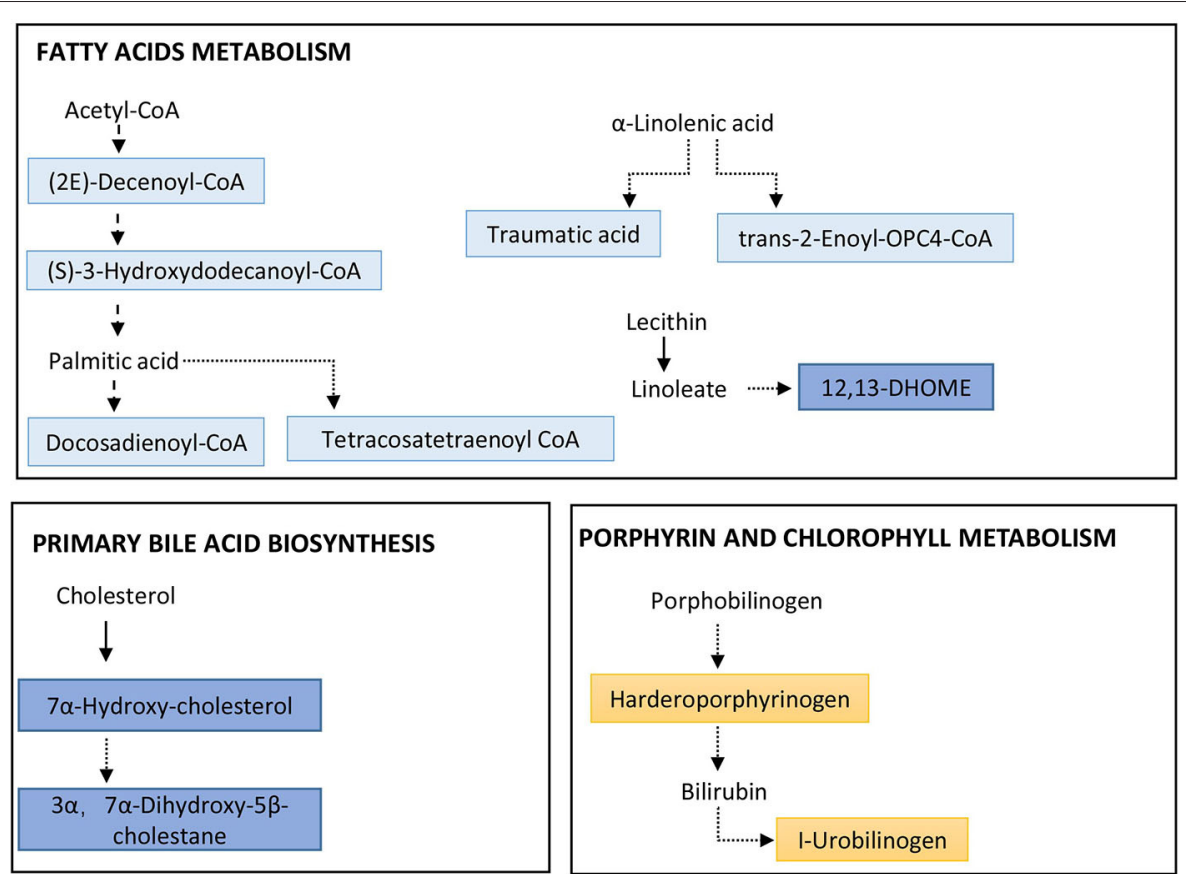

\section{CYSTEINE AND METHIONINE METABOLISM}
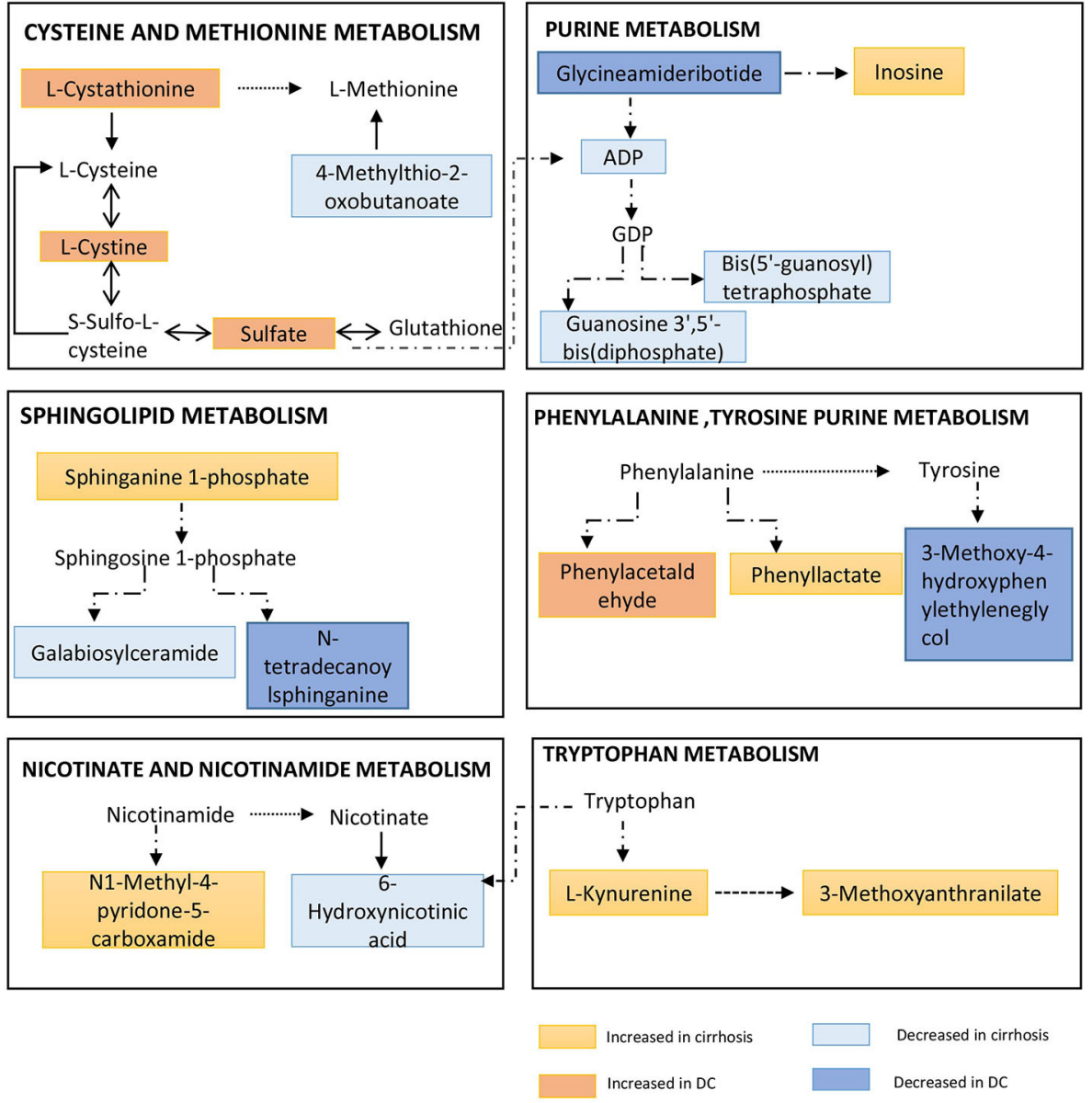

FIGURE 4 | Pathway enrichment of metabolites in metabolome fingerprint. A typical feature is that a series of metabolites related to long-chain fatty acids (e.g., 12,13-DHOME, trans-2-Enoyl-OPC4-CoA and Docosadienoyl-CoA, etc.) are significantly reduced. And, a key feature of decompensated liver cirrhosis was observed to be used as energy supply by proteolysis (incomplete breakdown products of protein catabolism increased significantly in DC group). 
L-kynurenine is associated with the presence of inflammation and energy expenditure (7).

We also observed significant decrease of 7alphahydroxycholesterol (the catalyzing product of Cyp7a1) in decompensated autoimmune hepatic cirrhosis (30). Previous studies have shown that Cyp7al is positively associated with liver inflammation $(31,32)$. Cyp7a1 is the rate-limiting enzyme in the "classic pathway" of bile acids synthesis. The reduction of bile acid synthesis will inevitably cause the dynamic balance of enterohepatic circulation, which will lead to persistent inflammation in the liver (33). In addition, bile acids can help emulsify fat, enhance lipolysis, and improve the solubility of lipids by forming mixed micelles, promote the absorption of lipids in the intestine, and improve lipolysis. And, the interaction between bile acids and intestinal microbiota is not unidirectional. It is necessary to study the intestinal bacteria of AIH patients to elucidate the potential impact of gut microbial on bile acids metabolism and its further effects on liver immune and metabolic microenvironments. Interestingly, the metabolites related to long-chain fatty acids in the metabolic fingerprint of cirrhosis are all reduced, which indicate that $\beta$-oxidation of fatty acids is enhanced, and the body needs to consume a lot of energy for immune response and immune cell activation and proliferation (34). There are many proteins with incomplete catabolism in the fingerprint of decompensated metabolism. These findings imply that proteolysis may increase due to energy requirements. In previous reports, the skeletal muscles of rats have a significant accumulation of sphinganine and sphingosine after exercise, which may be related to the increase in energy requirements. Furthermore, ceramide promotes apoptosis whereas sphingosine-1-phosphate can inhibit apoptosis and induce cell growth. So, the reaction will tend to transform to sphingosine. Therefore, Galabiosyl ceramide and $\mathrm{N}$-tetradecanoyl sphinganine will decrease (35).

Based on the favorable aspects of metabolic reprogramming mechanisms of $\mathrm{AIH}$, we can explore the utility of characteristic metabolites to facilitate diagnosis or monitoring of disease progression. We therefore found that the intensity ratio of a pair of metabolites [Mesobilirubinogen and 6-Hydroxynicotinic acid] can be applied to recognizing cirrhosis in AIH patients; furthermore, the paired metabolites $[\operatorname{LysoPA}(8: 0 / 0: 0)$ and 7alpha-Hydroxycholesterol] can predict the development of decompensated cirrhosis for AIH. Since the intensity ratio of paired metabolites can eliminate the system bias of instruments in different laboratories, these metabolite pairs can be much easier to be applied in clinical settings. Compared with targeted quantitation of metabolites by mass spectrometry, the intensity ratio of paired metabolites is much cheaper and simpler (36).

\section{REFERENCES}

1. Giorgina MV, Diego V, Albert JC, Michael PM, Edward LK, John MV. Autoimmune hepatitis. Nat Rev Dis Primers. (2018) 4:18018. doi: 10.1038/nrdp.2018.18

2. Mieli-Vergani G, Vergani D, Czaja AJ, Manns MP, Krawitt EL, Vierling JM, et al. Autoimmune hepatitis. Nat Rev Dis Primers. (2018) 4:18017. doi: $10.1038 /$ nrdp. 2018.17
In summary, liver metabolic dysfunction featuring enhanced lipolysis and increased proteolysis, may play an important role in the pathogenesis and progression of liver cirrhosis in AIH patients. The established metabolic fingerprint profile related to liver cirrhosis and decompensation can be used as a resource for metabolic adaptation and metabolic reprogramming of $\mathrm{AIH}$, and provide guidance for future clinical prognosis, mechanisms, and new treatment research. Furthermore, this finding suggests that metabolic reprogramming may be a new direction in treating $\mathrm{AIH}$.

\section{DATA AVAILABILITY STATEMENT}

The original contributions presented in the study are included in the article/Supplementary Material, further inquiries can be directed to the corresponding authors.

\section{ETHICS STATEMENT}

The studies involving human participants were reviewed and approved by Medical Ethics Committee of Fifth Medical Center of Chinese PLA General Hospital. The patients/participants provided their written informed consent to participate in this study.

\section{AUTHOR CONTRIBUTIONS}

J-bW, Y-cY, and Z-sZ were responsible for the study concept and design. S-sL, JJ, S-sC, YH, ZS, G-ZS, and XH performed sample collection, conducted LC-MS data analysis, and manuscript preparation. S-sL and J-bW drafted the manuscript. Z-tZ, H-zZ, Z-sZ, and Y-cY reviewed and modified the manuscript. MN helped with data analysis. All authors contributed to the article and approved the submitted version.

\section{FUNDING}

This study was supported by the grants from the National Natural Science Foundation of China (Nos. 82074112 and 81721002) to J-bW and X-hX, respectively; and the Young Talent Incubating Project of Chinese PLA General Hospital (Nos. 2019-JQPY-003) to J-bW.

\section{SUPPLEMENTARY MATERIAL}

The Supplementary Material for this article can be found online at: https://www.frontiersin.org/articles/10.3389/fmed. 2021.644376/full\#supplementary-material

3. Pape S, Schramm C, Gevers TJ. Clinical management of autoimmune hepatitis. United European Gastroenterol J. (2019) 7:1156-63. doi: 10.1177/2050640619872408

4. Liberal R, Grant CR. Cirrhosis and autoimmune liver disease: current understanding. World J Hepatol. (2016) 8:115768. doi: 10.4254/wjh.v8.i28.1157

5. Czaja AJ. Diagnosis and management of autoimmune hepatitis: current status and future directions. Gut Liver. (2016) 10:177. doi: 10.5009/gnl15352 
6. Sucher E, Sucher R, Gradistanac T, Brandacher G, Schneeberger S, Berg T. Autoimmune hepatitis-immunologically triggered liver pathogenesis-diagnostic and therapeutic strategies. J Immunol Res. (2019) 2019:9437043. doi: 10.1155/2019/9437043

7. Clària J, Moreau R, Fenaille F, Amorós A, Junot C, Gronbaek H, et al. Orchestration of tryptophan-kynurenine pathway, acute decompensation, and acute-on-chronic liver failure in cirrhosis. Hepatology. (2019) 69:1686701. doi: 10.1002/hep.30363

8. Trefts E, Gannon M, Wasserman DH. The liver. Curr Biol. (2017) 27: R114751. doi: 10.1016/j.cub.2017.09.019

9. Bujak R, Struck-Lewicka W, Markuszewski MJ, Kaliszan R. Metabolomics for laboratory diagnostics. J Pharm Biomed Anal. (2015) 113:10820. doi: 10.1016/j.jpba.2014.12.017

10. Yu M, Zhu Y, Cong Q, Wu C. Metabonomics research progress on liver diseases. Can J Gastroenterol Hepatol. (2017) 2017:8467192. doi: 10.1155/2017/8467192

11. Zhou C, Jia HM, Liu YT, Yu M, Chang X, Ba YM, et al. Metabolism of glycerophospholipid, bile acid and retinol is correlated with the early outcomes of autoimmune hepatitis. Mol bioSyst. (2016) 12:157485. doi: 10.1039/C6MB00092D

12. Wang JB, Pu SB, Sun Y, Li ZF, Niu M, Yan XZ, et al. Metabolomic profiling of autoimmune hepatitis: the diagnostic utility of nuclear magnetic resonance spectroscopy. J Proteome Res. (2014) 13:3792-801. doi: 10.1021/pr50 $0462 \mathrm{f}$

13. Lytton SD, Osiecki M, MałgorzataWozniak, Cukrowska B, Wierzbicka A, Goliszek M, Socha P, et al. Tryptophan-kynurenine profile in pediatric autoimmune hepatitis. Immunol Res. (2019) 67:39-47. doi: 10.1007/s12026-019-9068-1

14. EASL Clinical Practice Guidelines: autoimmune hepatitis. J Hepatol. (2015) 63:971-1004. doi: 10.1016/j.jhep.2015.06.030

15. Moreau R, Clària J, Aguilar F, Fenaille F, Lozano JJ, Junot C, et al. Blood metabolomics uncovers inflammation-associated mitochondrial dysfunction as a potential mechanism underlying ACLF. J Hepatol. (2020) 72:688701. doi: 10.1016/j.jhep.2019.11.009

16. Zhang L, Niu M, Wei AW, Tang JF, Tu C, Bai ZF, et al. Risk profiling using metabolomic characteristics for susceptible individuals of drug-induced liver injury caused by Polygonum multiflorum. Arch Toxicol. (2020) 94:24556. doi: 10.1007/s00204-019-02595-3

17. Czaja AJ. Diagnosis and management of autoimmune hepatitis. Clin Liver Dis. (2015) 19:57-79. doi: 10.1016/j.cld.2014.09.004

18. Mack CL, Adams D, Assis DN, Kerkar N, Manns MP, Mayo MJ, et al. Diagnosis and management of autoimmune hepatitis in adults and children: 2019 practice guidance and guidelines from the American Association for the study of liver diseases. Hepatology. (2020) 72:671-722. doi: 10.1002/hep. 31065

19. Song Y, Yang H, Lin L, Jiang K, Liu WT, Wang BM, et al. [Albuminto-bilirubin scores for assessing the prognosis in autoimmune hepatitisrelated cirrhosis]. Zhonghua Gan Zang Bing Za Zhi. (2019) 27:7726. doi: 10.3760/cma.j.issn.1007-3418.2019.10.007

20. Lynch KD, Chapman RW, Keshav S, Montano-Loza AJ, Mason $\mathrm{AL}$, Kremer $\mathrm{AE}$, et al. Effects of vedolizumab in patients with primary sclerosing cholangitis and inflammatory bowel diseases. Clin Gastroenterol Hepatol. (2020) 18:179-87.e6. doi: 10.1016/j.cgh.2019. 05.013

21. Trauner M, Gulamhusein A, Hameed B, Caldwell S, Shiffman ML, Landis C, et al. The nonsteroidal farnesoid X receptor agonist cilofexor (GS-9674) improves markers of cholestasis and liver injury in patients with primary sclerosing cholangitis. Hepatology. (2019) 70:788-801. doi: 10.1002/hep. 30509
22. Li Q, Lu C, Li W, Huang Y, Chen L. The gamma-glutamyl transpeptidase-toalbumin ratio predicts significant fibrosis and cirrhosis in chronic hepatitis B patients. J Viral Hepat. (2017) 24:1143-50. doi: 10.1111/jvh.12751

23. Liu L, Lan Q, Lin L, Lu J, Ye C, Tao Q, et al. Gamma-glutamyl transpeptidaseto-platelet ratio predicts the prognosis in HBV-associated acute-on-chronic liver failure. Clin Chim Acta. (2018) 476:92-7. doi: 10.1016/j.cca.2017.11.017

24. Brahim I, Brahim I, Hazime R, Admou B. [Autoimmune hepatitis: immunological diagnosis]. Presse Med. (2017) 46:100819. doi: 10.1016/j.lpm.2017.08.012

25. Gatza E, Wahl DR, Opipari AW, Sundberg TB, Reddy P, Liu C, et al. Manipulating the bioenergetics of alloreactive $\mathrm{T}$ cells causes their selective apoptosis and arrests graft-versus-host disease. Sci Transl Med. (2011) 3:67ra8. doi: 10.1126/scitranslmed.3001975

26. Ito $\mathrm{K}$, Ito $\mathrm{K}$. Metabolism and the control of cell fate decisions and stem cell renewal. Annu Rev Cell Dev Biol. (2016) 32:399409. doi: 10.1146/annurev-cellbio-111315-125134

27. Vander Heiden MG, DeBerardinis RJ. Understanding the intersections between metabolism and cancer biology. Cell. (2017) 168:657-69. doi: 10.1016/j.cell.2016.12.039

28. Cervenka I, Agudelo LZ, Ruas JL. Kynurenines: tryptophan's metabolites in exercise, inflammation, and mental health. Science. (2017) 357:eaaf9794. doi: 10.1126/science.aaf9794

29. Yin J, Ren W, Yang G, Duan J, Huang X, Fang R, et al. L-Cysteine metabolism and its nutritional implications. Mol Nutr Food Res. (2016) 60:134-46. doi: 10.1002/mnfr.201500031

30. Liu H, Pathak P, Boehme S, Chiang JY. Cholesterol $7 \alpha$-hydroxylase protects the liver from inflammation and fibrosis by maintaining cholesterol homeostasis. J Lipid Res. (2016) 57:1831-44. doi: 10.1194/jlr.M069807

31. Chambers KF, Day PE, Aboufarrag HT, Kroon PA. Polyphenol effects on cholesterol metabolism via bile acid biosynthesis, CYP7A1: a review. Nutrients. (2019) 11:2588. doi: 10.3390/nu11112588

32. Ferrell JM, Pathak P, Boehme S, Gilliland T, Chiang JYL. Deficiency of both farnesoid X Receptor and Takeda G protein-coupled receptor 5 exacerbated liver fibrosis in mice. Hepatology. (2019) 70:955-70. doi: 10.1002/hep.30513

33. Hartmann P, Chu H, Duan Y, Schnabl B. Gut microbiota in liver disease: too much is harmful, nothing at all is not helpful either. Am J Physiol Gastrointest Liver Physiol. (2019) 316:G563-G573. doi: 10.1152/ajpgi.00370.2018

34. Baranowski M, Błachnio-Zabielska AU, Charmas M, Helge JW, Dela F, Ksiazek M, et al. Exercise increases sphingoid base-1-phosphate levels in human blood and skeletal muscle in a time- and intensity-dependent manner. Eur J Appl Physiol. (2015) 115:993-1003. doi: 10.1007/s00421-014-3080-x

35. Nguyen P, Leray V, Diez M, Serisier S, Le Bloc'h J, Siliart B, et al. Liver lipid metabolism. J Anim Physiol Anim Nutr (Berl). (2008) 92:27283. doi: 10.1111/j.1439-0396.2007.00752.x

36. Ribbenstedt A, Ziarrusta H, Benskin JP. Development, characterization and comparisons of targeted and non-targeted metabolomics methods. PLOS ONE. (2018) 13:e0207082. doi: 10.1371/journal.pone.0207082

Conflict of Interest: The authors declare that the research was conducted in the absence of any commercial or financial relationships that could be construed as a potential conflict of interest.

Copyright (C) 2021 Li, Niu, Jing, Huang, Zhang, Chen, Shi, He, Zhang, Xiao, Zou, Yu and Wang. This is an open-access article distributed under the terms of the Creative Commons Attribution License (CC BY). The use, distribution or reproduction in other forums is permitted, provided the original author(s) and the copyright owner(s) are credited and that the original publication in this journal is cited, in accordance with accepted academic practice. No use, distribution or reproduction is permitted which does not comply with these terms. 